\title{
DISKS AND OUTFLOWS AS SEEN FROM THE IRAM INTERFEROMETER
}

\author{
S. GUILLOTEAU, A. DUTREY AND F. GUETH \\ IRAM, 300 rue de la piscine \\ F-38406 Saint Martin d'Hères Cedex, FRANCE
}

\begin{abstract}
At-stract. The IRAM interferometer has been used to map 3 high velocity outflows from Class 0 sources in $\mathrm{SiO}$ and $\mathrm{CO}$ rotational lines. The images show that such outflows are driven by highly collimated, dense, but optically invisible jets, through large travelling bow shocks. Precession is important in explaining some morphological and kinematic aspects. A significant part of the $\mathrm{SiO}$ emission is directly associated with the jet. Images of the molecular core surrounding L 1157 in CO isotopic lines show direct evidence for infall in a large, flattened disk.

For more evolved objects, "surveys" of mm emission from T Tauri stars provide evidence for circumstellar dust disks with relatively flat surface density distribution and typical radii 100 to $200 \mathrm{AU}$. Much larger gas disks (radii 800 to $1000 \mathrm{AU}$ ) have been detected and imaged in a few objects ( 2 singles and 2 binaries) through $\mathrm{CO}$ isotopomers, but in most sources ${ }^{13} \mathrm{CO}$ is difficult to detect. The spectral line images provide unambiguous evidence for Keplerian rotation. In two cases (GG Tau and DM Tau), direct measurements of the gas mass (independently of the dust) has been made thanks to the detection of 7 molecular species. The molecules are found to be depleted by factors between 5 (for $\mathrm{CO}$ ) to 100 (for $\mathrm{H}_{2} \mathrm{CO}$ ). These results suggest that circumstellar disks consist in a small, dense, inner disk of outer radius $\simeq 150 \mathrm{AU}$ (traced by the dust emission), surrounded by a much larger, less massive, outer disk (traced by the gas emission).
\end{abstract}

\section{Introduction}

Although molecular outflows have been known since 1980, they still represent an observational challenge. Many low resolution studies have been performed, but very few high angular resolution images exist because of the 
combination of 3 properties: long linear sizes, high collimation (implying narrow structures), and relatively low brightness for most molecular lines. With its large antennas, good site and low noise receivers, the IRAM interferometer has become the instrument of choice to study outflows since the development of new mosaicing techniques involving joint deconvolution of overlapping fields (Gueth et al., 1995). Note that mosaicing is specially suited for elongated objects like outflows, while for rounder objects it would be nearly as efficient to simply use an array with smaller antennas.

When dealing with circumstellar disks around T Tauri stars, the sensitivity problem is even more critical, since the expected line widths are much smaller than for outflows, and the sizes very small. The IRAM interferometer provide unsurpassed performances at $1.3 \mathrm{~mm}$, where the primary beam is well matched to the total extent of the disks. The winter conditions, with precipitable water vapor content frequently below $2 \mathrm{~mm}$ and average temperatures $-10^{\circ} \mathrm{C}$, provide excellent phase stability, with effective seeing below $0.3^{\prime \prime}$. With the baseline extension (Sep. 1995) up to $408 \mathrm{~m}$ length, observations with $0.5^{\prime \prime}$ resolution have become possible.

This paper presents an overview of research going on at IRAM on high velocity outflows from Class 0 objects and their exciting sources, and on disks around $\mathrm{T}$ Tauri stars.

\section{Outflows from Class 0 objects}

\subsection{1448}

The first highly collimated outflow studied with the IRAM interferometer is $\mathrm{L} 1448$. The inner part of the flow was observed in the $\mathrm{SiO}(2-1)$ transition, with $2^{\prime \prime}$ resolution (Guilloteau et al., 1992). These observations revealed that the high degree of collimation and the acceleration of the gas was essentially achieved within $1000 \mathrm{AU}$ of the exciting star. However, SiO, because of its short lifetime and high critical density, is a very selective tracer and does not represent the bulk of the gas. Subsequent observations of the $\mathrm{CO}(1-0)$ line (Bachiller et al., 1995) clearly revealed the outflow cavity walls. Surprisingly, the red and blue lobes present different opening angles close to the star. The picture is complicated by the possibility of a direct collision of the blue lobe with the red lobe of the outflow originating near L1448-IRS3. This collision provides a possible explanation for the strong shocks revealed in $\mathrm{H}_{2}$ and for the general bending of the blue lobe.

To further study the molecular "bullets", which remained only marginally detected in the $\mathrm{CO}$ image, the complete red lobe has been mapped in $\mathrm{SiO}(2-1)$ using a 5-field mosaic (Dutrey et al., 1997a). The SiO emission is clearly confined in a jet-like structure, well aligned with the CO cavity axis, and terminating in a strong bow shock. More detailed analysis of the 

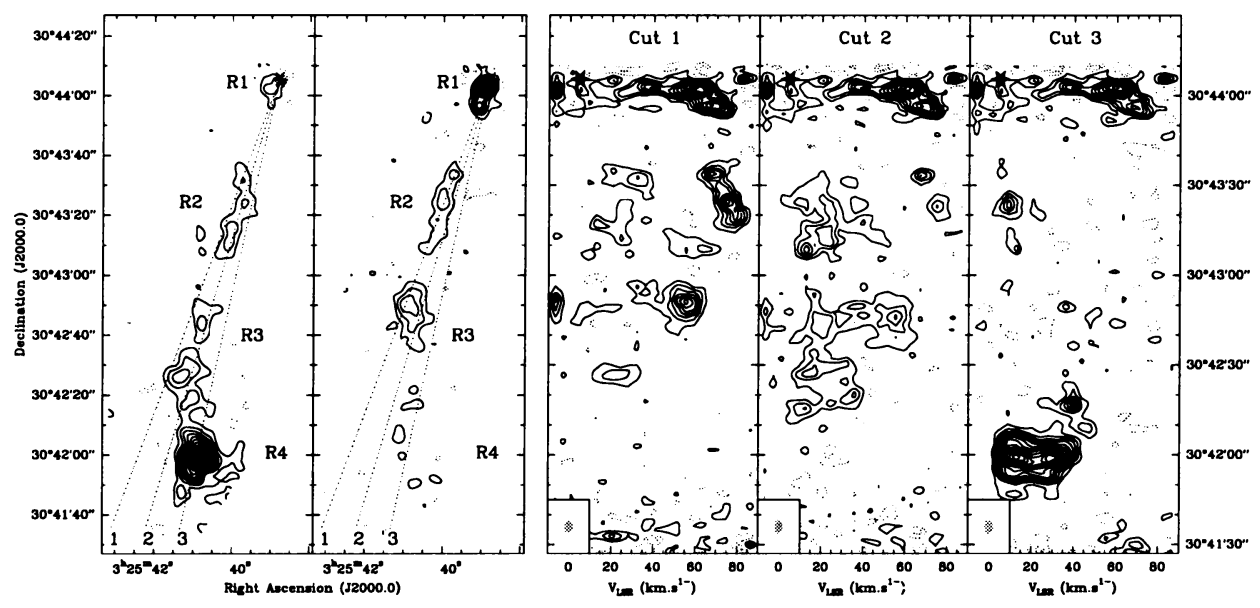

Figure 1. Left: Maps of the $\mathrm{SiO} v=0 \mathrm{~J}=2-1$ emission associated with the red lobe of L1448-mm. The intensity is integrated from -7 to $34 \mathrm{~km} \cdot \mathrm{s}^{-1}$ (left panel) and 35 to $80 \mathrm{~km} . \mathrm{s}^{-1}$ (center panel). The beam is $4.2^{\prime \prime} \times 3.4^{\prime \prime}$ at P.A. $62^{\circ}$. Contour step is 0.5 $\mathrm{Jy} / \mathrm{beam} . \mathrm{km} . \mathrm{s}^{-1}$ or $0.56 \mathrm{~K} . \mathrm{km} \cdot \mathrm{s}^{-1}$ in both maps. Right: position-velocity diagram along the lines 1-2-3. First contour and step are $20 \mathrm{mJy}$ or $0.23 \mathrm{~K}$. From Dutrey et al. 1997a

spatio-kinematic information reveals that most of the $\mathrm{SiO}$ emission actually originates in a few large, clumpy, bow shocks. The projected velocities of the shocks decrease with distance from the exciting source. The jet appears to wander ("precess") by about $10^{\circ}$, but may also have been deflected against a dense molecular clump (see Fig.1).

The L 1448 example shows that jet-driven flow models may be successful in explaining molecular outflows. However, even in such a young flow, the influence of the surrounding medium has made the picture so complex that it is difficult to separate intrinsic processes (e.g. precession) from external influences (e.g. deflection). Searching for a less confused case is thus necessary.

\section{2. $\mathrm{L} 1157$}

Compared to L 1448, L 1157 offers the double advantage of being isolated and at high declination.

The CO emission from the blue-shifted lobe of L 1157 was reconstructed from a 10-field mosaic; inclusion of short spacing information from the 30-m telescope was essential to recover the complete picture (Gueth et al., 1996). The CO emission (Fig. 2) clearly delineates two well-defined cavities, with projected axes separated by about $6^{\circ}$. Moreover, the kinematics can be simply explained by a precessing jet model in which the "terminal" cavity $\mathrm{C} 1$ is nearly in the plane of the sky, while the brighter cavity C2 is about 


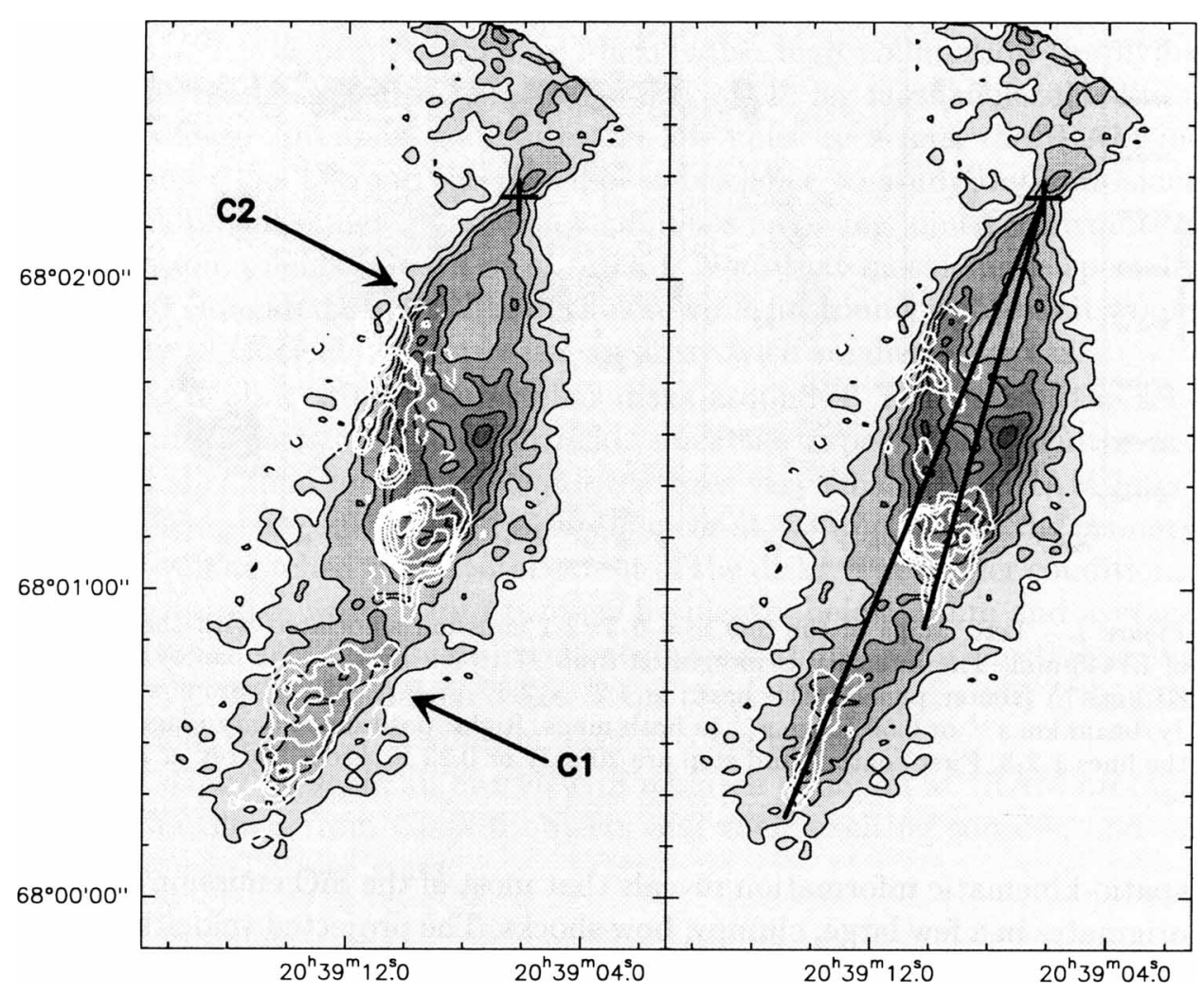

Figure 2. Left: integrated $\mathrm{CO}$ (greyscale) and $\mathrm{SiO}$ emissions (white contours) in the blue-shifted lobe of L 1157. Right: $\mathrm{SiO}$ emission at $2.8 \mathrm{~km} \cdot \mathrm{s}^{-1}$ superimposed on the integrated CO emission. Angular resolution is about $3^{\prime \prime}$ (Gueth et al. 1997a)

$10^{\circ}$ from the plane of the sky towards us. The model also explains the apparent asymmetries in the brightness distribution.

The $\mathrm{SiO}$ image fully confirms this model, since $\mathrm{SiO}$ is visible along the cavity walls which are directly exposed to the precessing jet: western edge of the $\mathrm{C} 1$ cavity, and eastern edge of the C2 cavity (Gueth et al., 1997a), see Figure 2. A very strong "shock" is visible at the apex of the C2 cavity, at a position where, according to the precession model derived from the $\mathrm{CO}$ observations, the driving jet crosses the wall of the $\mathrm{C} 1$ cavity. The $\mathrm{CO}$ emission of the $\mathrm{C} 2$ cavity is clearly in the wake of this strong $\mathrm{SiO}$ shock. These positional differences between $\mathrm{SiO}$ and $\mathrm{CO}$ emissions is a strong argument in favor of jet-driven flow models.

Linear features whose axes intersect exactly on the continuum emission marking the exciting source of L 1157 are well identified, but contrary to $\mathrm{L} 1448$, the $\mathrm{SiO}$ emission does not clearly delineate a jet. Moreover, al- 
though the strong $\mathrm{SiO}$ emission at the $\mathrm{C} 2$ cavity apex has a clear bow-like appearance, its kinematics does not agree with simple bow shock models. The very well-defined linear structure just ahead of the $\mathrm{C} 2$ apex is intriguing: since $\mathrm{SiO}$ is presumably formed in the shock, it is a priori surprising to find emission ahead of the shock front. This structure may be a magnetic precursor or a nose cone preceeding the main shock front. Alternatively, it could be that $\mathrm{SiO}$ is already present in the jet, perhaps created by oblique shocks in the jet boundary layer. Note that a similar, though less clear, linear "precursor" is visible ahead of the terminal bow shock of L 1448.

\section{3. $\mathrm{HH} 211$}

Despite their differences, the examples presented above strongly suggest that young molecular outflows may be driven by supersonic jets. However, the jets remain elusive, being revealed only by secondary indicators like $\mathrm{SiO}$.

HH 211 is an IR discovered outflow (McCaughrean et al., 1994), probably nearly in the plane of the sky as suggested by the small extinction difference between both lobes. It is likely to be extremely young, and may offer a unique opportunity to study the earliest phases of the outflow processes, perhaps before external influences blurr the picture.

We have performed a complete 9-field $\mathrm{CO}(2-1)$ mosaic of this jet, with $1.5^{\prime \prime}$ angular resolution (Gueth and Guilloteau, 1997). Compared to L 1448 and L 1157, HH 211 is full of surprises (see Fig.3):

- Low velocity CO delineates the cavity walls (as expected) but coincides with the $\mathrm{H}_{2}$ emission (in $\mathrm{L} 1448$ and $\mathrm{L} 1157 \mathrm{H}_{2}$ emission is more closely related to the $\mathrm{SiO}$ features, although detailed comparisons are hampered by astrometric problems in the $\mathrm{H}_{2}$ images).

- High velocity CO traces a wonderful, narrow, jet, which terminates exactly at the position of the $\mathrm{H}_{2}$ emission (Fig.3)

- The velocity law is almost perfectly linear with distance from the star ("Hubble" law).

- Accounting for the low inclination, the deprojected velocity reaches at least $200 \mathrm{~km} . \mathrm{s}^{-1}$. Hence, the jet is very young (500 years).

- A direct estimate of the mean jet density is possible from the CO column density and apparent sizes: the $\mathrm{CO}$ jet is dense $\left(\mathrm{n}\left(\mathrm{H}_{2}\right)>\right.$ $10^{5} \mathrm{~cm}^{-3}$ ).

- The low velocity cavity shape is well reproduced by travelling bow shock models.

- There is a well resolved, flattened continuum source at the jet basis, and it is elongated nearly perpendicular to the jet.

The CO observations also suggest a preceding outflow episode whose 


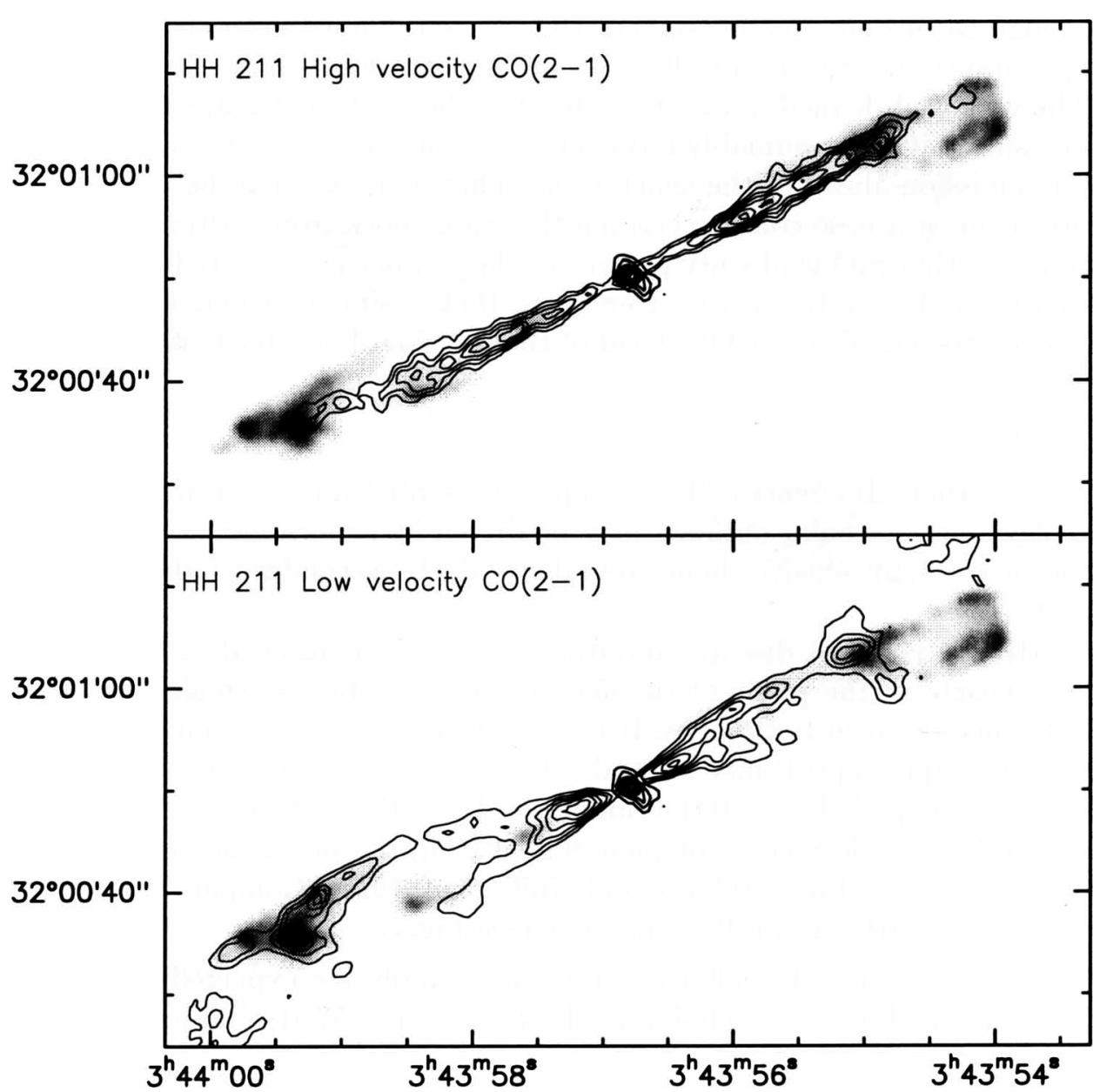

Figure 3. Contour map of the high and low velocity ${ }^{12} \mathrm{CO}(2-1)$ emission in $\mathrm{HH} 211$, superimposed on the $\mathrm{H}_{2} 2.2 \mu \mathrm{m}$ image in greyscale from McCaughrean et al. (1994). The thick contours indicate the $1.3 \mathrm{~mm}$ continuum emission. Angular resolution is $1.4 \times 1.1^{\prime \prime}$ at PA $83^{\circ}$.

axis is tilted by a few degrees from the current jet axis. HH 211 is also a strong $\mathrm{SiO}$ source. Interferometric observations in $\mathrm{SiO}(2-1)$ (Gueth et al., 1997b), and $\mathrm{SiO}(1-0)$ (Chandler and Richer, 1997) show that $\mathrm{SiO}$ is only present in the jet. This is a striking difference with L 1448 and L 1157.

As such, HH 211 is an ideal case for very young jet-driven flows. The existence of sufficiently strong $\mathrm{CO}$ emission from the jet will allow much better determination of its physical parameters (density, temperature, momentum) than would be possible using any other molecular tracer, since, contrary for example to $\mathrm{SiO}$ or $\mathrm{NH}_{3}$, the $\mathrm{CO}$ abundance is not affected by 
variations in the chemistry.

\subsection{CONCLUSIONS}

From the above examples, a few general properties of outflows from Class 0 sources can be derived. First, jets are dense, with mean densities of at least $10^{5} \mathrm{~cm}^{-3}$. Second, the natural opening angles for the cavities of jet driven flows is around $30-40^{\circ}$. Third, precession (or jet wandering) may account for about $10^{\circ}$ in the opening angle of the outflow cavities, but not more. Fourth, shock structure is complex, with small linear precursors ahead of the most striking bow shocks. However, the variety of characters and the importance of the surrounding medium should be stressed. Only 3 objects have been studied so far, but all present very different aspects.

The above conclusions should not be generalized to all outflows. In the case of L1551-IRS5, the outflow cavity is apparently widely opened. Whether this results from an age difference or from the different characteristics of the star formation processes in the Taurus region is entirely open. More systematic studies (e.g. small surveys) would be required to properly address this problem.

\section{Infalling envelopes ?}

As mentioned above, a resolved continuum source lies at the origin of the outflow in HH 211. L 1448 also presents a compact continuum source at the base of the outflow (Bachiller et al., 1995). Among the sources discussed above, only L 1157 has been studied in details so far, and the situation is here somewhat more complex. Although a compact core exists, and is elongated perpendicular to the flow, a large fraction of the $2.7 \mathrm{~mm}$ continuum flux is spread over 10-15" (5000 AU) (Gueth et al., 1997c). This extended envelope actually contains most of the mass. In addition, the images show that the continuum emission is brighter along the edges of the outflow cavity (Fig.4), suggesting that the outflow has been energetic enough to affect a significant fraction of the envelope mass. Spectral line observations also reveal that the continuum (dust) core and $\mathrm{C}^{18} \mathrm{O}(1-0)$ data are nearly coextensive, while ${ }^{13} \mathrm{CO}(1-0)$ is clearly visible at the edge of the outflow.

The spectra reveal a deep, redshifted, self-absorption feature in ${ }^{13} \mathrm{CO}(1-$ 0 ) towards the dust core, strongly suggesting infall motions. Note that in general, interferometric observations can easily produce "false" self-absorption features, because an interferometer resolves out the extended structures. To avoid such a bias, it is essential to include short spacing information (see Fig.5). Because ${ }^{13} \mathrm{CO}$ has a relatively low dipole moment and is easy to excite, the deep self-absorption feature observed in $\mathrm{L} 1157$ requires special conditions to be produced, namely a disk-like geometry with a large 


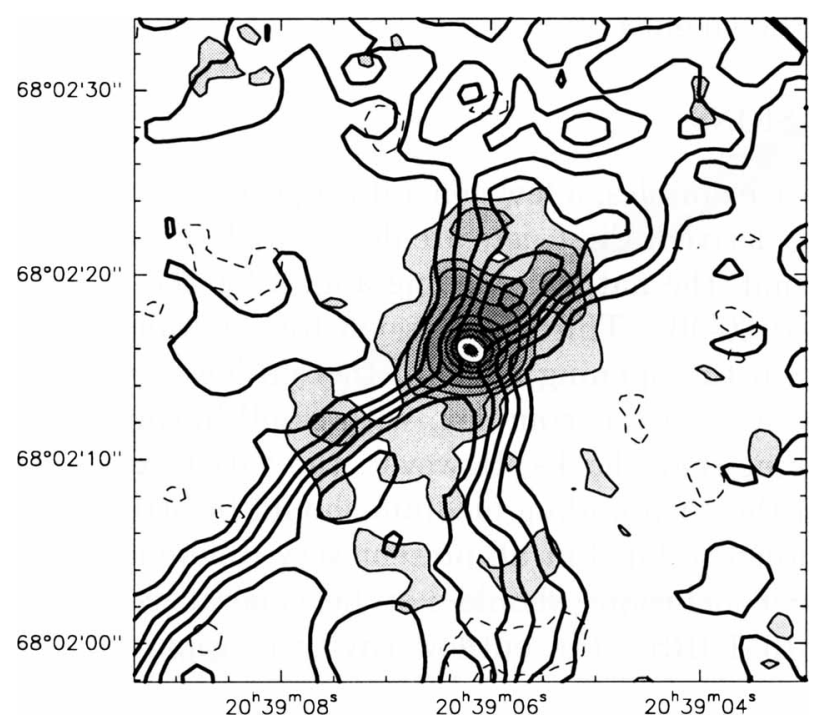

Figure 4. Overlay of the $\lambda 2.7 \mathrm{~mm}$ continuum emission (greyscale) and the integrated ${ }^{12} \mathrm{CO}(1-0)$ emission (thick contours). The extended component of the continuum emission is clearly associated with the edges of the molecular outflow. The thick white ellipse indicates the best fit gaussian model to the compact core of the continuum emission (Gueth et al., 1997c).

disk (radius $8000 \mathrm{AU}$ ) seen nearly edge-on. While such a situation is a priori unlikely, it is in agreement with the inclination derived for the outflow (Gueth et al., 1997c).

A common point to all 3 sources is that the diameters of the compact cores are about $1000 \mathrm{AU}$, although except for $\mathrm{HH} 211$ this is close to the resolution limit. Confirmation of these values by higher angular resolution observations would be important.

\section{Disks around $\mathbf{T}$ Tauri stars}

\subsection{DUST DISKS}

The diameters found for the dust cores in Class 0 objects are much larger than those found for $\mathrm{T}$ Tauri stars. In a survey of $2.7 \mathrm{~mm}$ emission in 33 $\mathrm{T}$ Tauri star systems, Dutrey et al. (1996) found that the emission from the stronger sources was marginally resolved, with angular sizes about $1^{\prime \prime}$. Interpreted in terms of disk models with power law surface density and temperature distributions, this suggests that circumstellar disks around $\mathrm{T}$ Tauri stars have a relatively flat density distribution, with outer radii around $150 \mathrm{AU}$. A similar conclusion was reached for HL Tau from sub- 


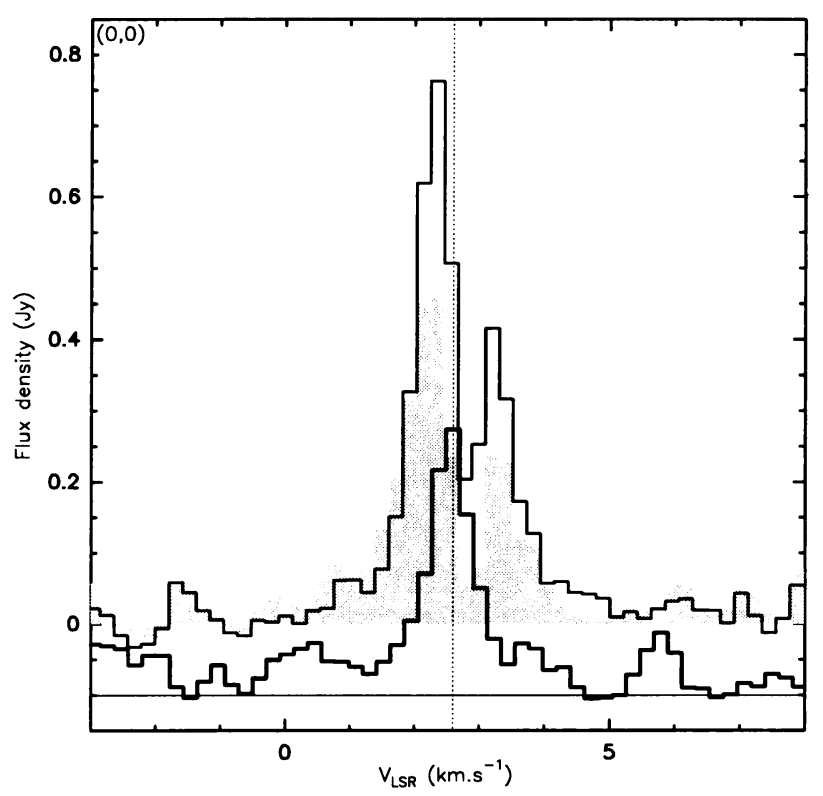

Figure 5. ${ }^{13} \mathrm{CO}(1-0)$ (thin line), $\mathrm{C}^{18} \mathrm{O}(1-0)$ (thick line) towards the mm source of $\mathrm{L} 1157$, showing the pronounced red-shifted self-absorption. The grey-scale histogram is the ${ }^{13} \mathrm{CO}(1-0)$ interferometric spectrum before the addition of short spacing information (Gueth et al., 1997c).

arcsecond resolution images from the BIMA array (Mundy et al., 1996). Comparison of the measured $2.7 \mathrm{~mm}$ flux densities with bolometer measurements at $1.3 \mathrm{~mm}$ and $0.8 \mathrm{~mm}$ indicate a mean dust emissivity spectral index $\beta \simeq 1.0$ (see Dutrey et al. 1996 and references therein).

However, interpretations based on single frequency size measurements are ambiguous, since the same angular size can be produced by various combinations of radial temperature and opacity distributions in the circumstellar disks. To remove this ambiguity, we have started a "mini-survey" of 12 $\mathrm{T}$ Tauri stars using the newly available dual-frequency capabilities and extended baselines $(400 \mathrm{~m})$ of the IRAM interferometer (Dutrey et al., 1997b). These new data have sub-arcsecond angular resolution, and also allow to measure simultaneously the angular sizes at 2.7 and $1.3 \mathrm{~mm}$. The preliminary results confirm the initial measurements. In particular, the sizes are identical at both frequencies, a property which is most easily explained if the brightness distributions are identical. Since the dust opacity at $1.3 \mathrm{~mm}$ is 2-3 times higher than at $2.7 \mathrm{~mm}$, this rules out steep surface density distributions, and also implies moderate dust opacities.

Another direct result of these angular size measurements is the apparent elongation and position angles of the dust disks. From the aspect ratio, a 
lower limit to the disk inclination (taking into account the influence of the seeing) can be derived.

\subsection{GAS DISKS KINEMATICS}

Large gas disks have been detected and imaged in 4 systems: GG Tau in ${ }^{13} \mathrm{CO}(1-0)$ (Dutrey et al., 1994), DM Tau in $\mathrm{CO}(1-0)$ (Guilloteau et al., 1997), GM Aur in ${ }^{12} \mathrm{CO}(2-1)$ (Dutrey et al., 1997c) and UY Aur in ${ }^{13} \mathrm{CO}(2-1)$ and (1-0) (Duvert et al., 1997). All 4 sources are either isolated from molecular clouds (GG Tau, DM Tau) or in regions of low extinction near the cloud edges (GM Aur, UY Aur).

Inclination and position angles of the dust disk can be compared to the morphology observed using a spectral line tracer. This has been done for ${ }^{12} \mathrm{CO}(2-1)$ in GM Aur (Dutrey et al., 1997c). A position angle of $55^{\circ} \pm 5^{\circ}$ and an inclination of $58^{\circ} \pm 5^{\circ}$ (0 meaning face on) are measured from the continuum emission. The kinematic pattern of the ${ }^{12} \mathrm{CO}(2-1)$ emission clearly indicates Keplerian rotation around the dust disk axis, and the derived position angle and inclination agree remarkably well (see Fig. 6). The quality of the ${ }^{12} \mathrm{CO}$ image is even sufficient to recover the radial temperature profile across the gaseous disk, and also the full 3-D orientation. Since the temperature decreases radially outwards, self-absorption effects are expected in an inclined flared (or geometrically thick) disk. This has been observed in GM Aur, and allows to indicate that the south-eastern part of the disk is closer to the observer than the north-western part.

Position angles, inclination and orientation measured with these $1.3 \mathrm{~mm}$ data are indeed in excellent agreement with those derived independently from the HST optical image (Stapelfeldt et al., 1997).

Keplerian rotation has also been found in GG Tau (Dutrey et al., 1994), DM Tau (Guilloteau et al., 1997), and UY Aur (Duvert et al., 1997). In GM Aur and GG Tau, the major axis defined by the spectral line emission coincides perfectly with that defined from the dust emission. In UY Aur, however, the continuum emission is too weak to allow to define a major axis independently from the $\mathrm{CO}$ pattern. Determination of Keplerian rotation requires the kinematics to be sampled by a sufficiently large number of channels (10 to 20), i.e. very high spectral resolution, and thus high sensitivity. Fig.7 illustrates the accuracy reached for UY Aur. Furthermore, it should be pointed out that it is essential that the images have a circular beam (or that the modelling incorporates properly the effective synthesized beam). Otherwise, the convolution by an elliptical beam can produce an apparent skewness in the velocity distribution which can easily be mis-interpreted as a combination of infall and rotation, specially when the kinematic pattern is insufficiently resolved. 
$4.25 \quad 5.75$

7.25

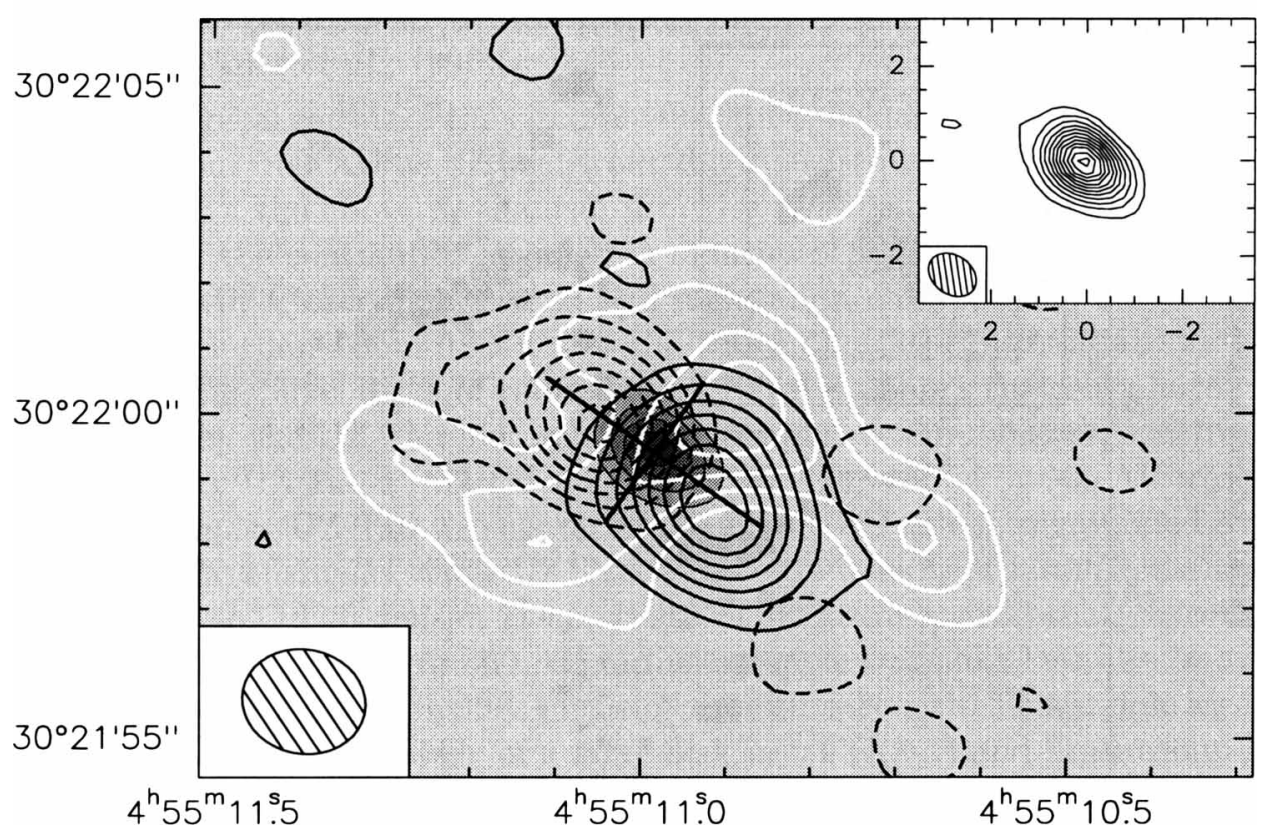

Figure 6. Contours of ${ }^{12} \mathrm{CO}(2-1)$ emission at 4.5, 5.75 (systemic), and $7.0 \mathrm{~km} / \mathrm{s}$ towards GM Aur, superposed on the continuum image at $1.3 \mathrm{~mm}$ in grey scale. The CO contour steps are $200 \mathrm{mJy} / \mathrm{beam}$. The cross indicates the continuum peak position, orientation and aspect ratio. Insert: higher resolution continuum image (synthesized beam $0.6^{\prime \prime} \times 0.7^{\prime \prime}$ at PA $56^{\circ}$ ); contour step is $7.5 \mathrm{mJy} /$ beam (from Dutrey et al. 1997c).

Both DM Tau and GM Aur are relatively old T Tauri stars $\left(\simeq 10^{7}\right.$ years). The kinematics of gas around younger objects like HL Tau does not seem dominated by Keplerian motions: infall has been suggested for HL Tau (Hayashi et al., 1993), although outflow motions make the picture ambiguous (Cabrit et al., 1996). This may suggest that Keplerian rotation is established relatively late in the evolution of circumstellar disks. On the other hand, GG Tau and UY Aur are very young $\left(<510^{5} \mathrm{yr}\right)$, but both are binary systems. Since binaries are more likely to form when the original specific angular momentum is large, it is perhaps not surprising to find Keplerian rotation established earlier in binary systems than in single stars. The evolutionary picture is also complicated by possible confusion with the surrounding medium (clouds, outflows). In this respect, it may be symptomatic that rotation has been unambiguously detected only in objects located in low extinction regions. 


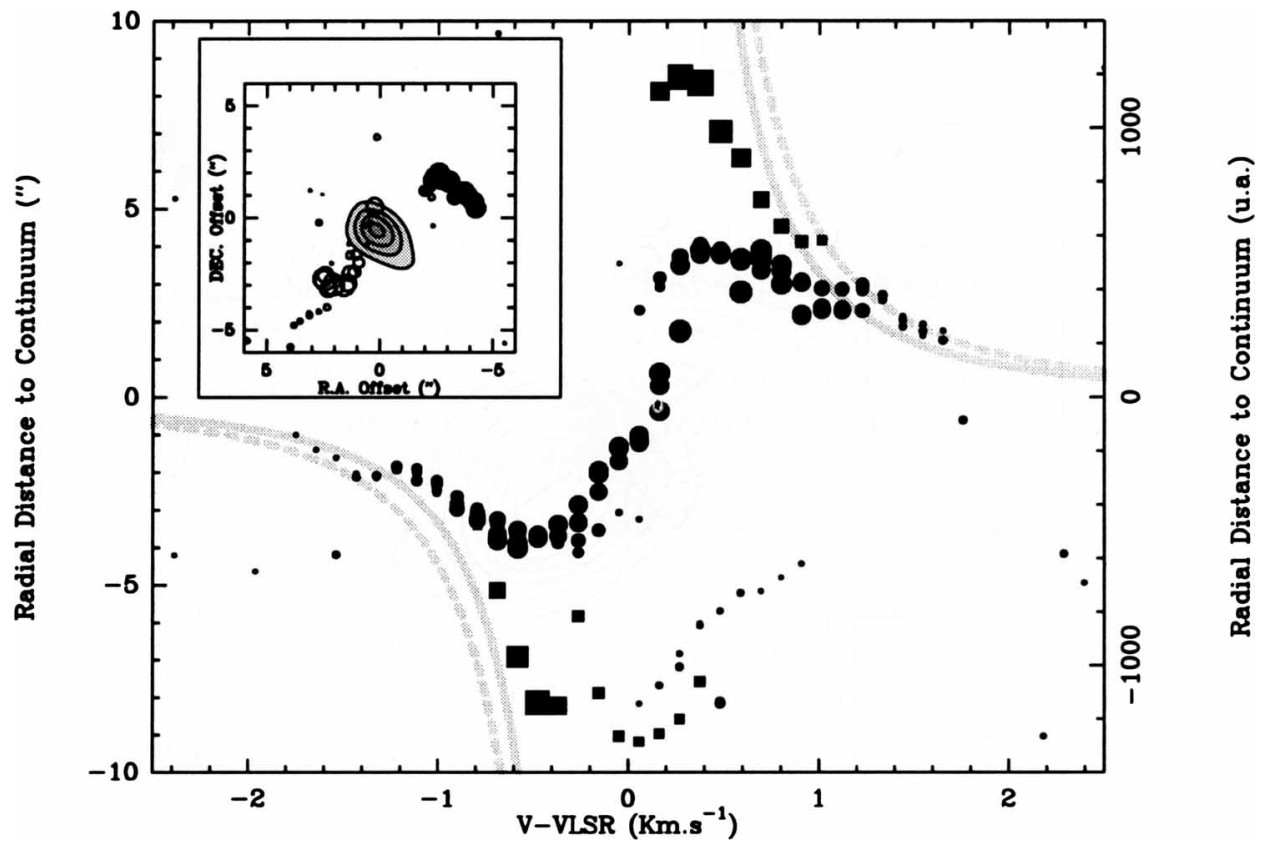

Figure 7. Velocity structure of the CO emission around the binary star UY Aur (separation $0.88^{\prime \prime}$ ). The radial distance of the centroid of emission is plotted against velocity. Squares are for ${ }^{13} \mathrm{CO}(1-0)$, circles for ${ }^{13} \mathrm{CO}(2-1)$. The marker sizes are proportional to the emission strength. The grey lines are Keplerian rotation curves for a central mass 0.7 $\mathrm{M}_{\odot}$, edge-on (dashed line) or inclined by $60^{\circ}$ (solid line). The inset shows the loci of the emission centroid with respect to the position of the $1.3 \mathrm{~mm}$ continuum emission (filled contours). Filled circles are for redshifted gas, empty circles for blueshifted gas (from Duvert et al. 1997).

\subsection{BINARIES}

More than half of all stars are members of binary systems. The influence of the binarity on the circumstellar environment is important. In the $2.7 \mathrm{~mm}$ survey of 33 systems, Dutrey et al. (1996) confirmed that single stars have on average stronger emission than binaries. GG Tau, the second strongest $\mathrm{mm}$ source among TTauri stars in Taurus, is the most flagrant counterexample to this general rule, but provides excellent clues on the origin of the difference. The mm emission from GG Tau was first resolved by Simon and Guilloteau (1992). Subsequent detailed mapping with $2^{\prime \prime}$ resolution indicated that the dust around GG Tau is actually forming a ring of inner radius $180 \mathrm{AU}$, inclined about $40^{\circ}$ from face on (Dutrey et al., 1994). The central part of this ring has presumably been cleared up by tidal interaction with the binary. Using the model from Artimowicz and Lubow (1994), Dutrey et al. (1994) predicted that the orbit of GG Tau was eccentric. 
These results have since been completely confirmed by IR adaptive optics images (Roddier et al., 1996).

If the typical radius of the circumstellar dust disk around single $\mathrm{T}$ Tauri stars is about $150 \mathrm{AU}$ as derived from the dual frequency survey (Dutrey et al., 1997b), the above mechanism provides a natural explanation for the weakness of the continuum emission in binaries of separations in the range 30 to $300 \mathrm{AU}$. Closer binaries can still maintain a circumbinary ring with radius larger than about $100 \mathrm{AU}$, while wider binaries can maintain individual circumstellar disks of radius up to $100 \mathrm{AU}$. This situation is nicely illustrated by the UZ Tau hierarchical quadruple system, where a spectroscopic binary UZ Tau-e (Matthieu et al., 1996) is separated by $500 \mathrm{AU}$ from the close binary UZ Tau-w (separation $50 \mathrm{AU}$ ). UZ Tau-e is surrounded by a disk typical of singles, while UZ Tau-w has only weak continuum emission (Dutrey et al., 1996; Jensen et al., 1996). This also suggests that gaseous disks, which extend out to $800 \mathrm{AU}$ radius in single stars like DM Tau, may exist around binaries regardless of the continuum strength. This simple idea actually led to the discovery of a large disk in ${ }^{13} \mathrm{CO}(2-1)$ and (1-0) around the binary UY Aur (separation 0.9"), see Fig.7 (Duvert et al., 1997).

\subsection{DISK MASSES FROM DUST}

The $\mathrm{mm}$ domain is the best frequency range to determine the total dust content of molecular disks, since the dust emission is optically thin, yet strong enough to be detectable over a useful range of masses. This was done by bolometer surveys near $1.3 \mathrm{~mm}$ for the Taurus region (Beckwith et al., 1990), and the interferometer data confirm that the optically thin assumption is valid for most of the disks.

For more distant star forming regions, the angular distances between stars become comparable to or smaller than the typical beams of bolometerequipped single-dish telescopes. Moreover, confusion with dust from the surrounding molecular clouds also become critical. Evidence for circumstellar disks has been demonstrated by the images of the proplyds in the Orion nebula (e.g. McCaughrean and O'Dell, 1996), but these observations provide little constraints on the average disk masses, since dust is easily opaque in the optical domain. Studies with $\mathrm{mm}$ interferometers are thus essential to properly measure the masses of circumstellar dust disks. However, because the flux density drops as the square of the distance, the sensitivity was a major problem, specially at $2.7 \mathrm{~mm}$ where most instruments operate (Mundy et al., 1995). Using the new $1.3 \mathrm{~mm}$ capabilities of the IRAM interferometer, Lada et al. (1996) have been able to detect 3 out of $\simeq 15$ proplyds or cm VLA sources in a field of the Orion Trapezium cluster. The estimated disk masses of the detected disks range from 0.007 to $0.016 \mathrm{M}_{\odot}$. 


\subsection{DISK MASSES FROM GAS}

For normal abundances and temperatures relevant to circumstellar disks (10-40 $\mathrm{K}$ at distances larger than $100 \mathrm{AU}$ from the star), the ${ }^{13} \mathrm{CO}(1-0)$ or (2-1) lines are much more optically thick than dust: the opacity ratio is of order 1000-2000 (Dutrey et al., 1996). Hence, it is surprising that only 4 systems out of 33 were detected in ${ }^{13} \mathrm{CO}(1-0)$ in the $2.7 \mathrm{~mm}$ survey (Dutrey et al., 1996). Several possibilities can be invoked to resolve the discrepancy: 1) the gas disks are small, 2) confusion with the ambient cloud dominates, 3 ) circumstellar disks are dominated by a compact central core of radius 100-200 AU, 4) molecules are strongly depleted, and 5) dust absorption coefficients are incorrect.

Proposition 1) is contradicted by the existence of large gas disks around GG Tau, GM Aur, DM Tau and UY Aur. Proposition 2) could be valid, since all previous sources are free of confusion. Size measurements do suggest that dust is indeed confined within $200 \mathrm{AU}$ from the star (for single stars), supporting proposition 3). Such a compact core would be optically thick in spectral lines but would escape detection because of beam dilution. A similar situation is advocated for the circumbinary ring of GG Tau (Dutrey et al., 1994), where modeling suggest that $90 \%$ of the mass is in a relatively narrow (width $<200 \mathrm{AU}$ ) ring. Using a standard dust absorption coefficient, molecular depletion was also found necessary to explain the weak isotopic CO lines, indicating that propositions 4) and 5) could be valid.

If neither the dust absorption coefficient, nor the molecular content of the gas disks are known, estimating the disk masses becomes a challenge. To address this key question, Dutrey, Guilloteau and Guélin (1997, DGG) have used a completely new approach. Using the 30-m telescope, they have searched for molecular lines with varying degrees of excitation in the DM Tau and GG Tau disks, and detected for the first time CN, HCN, $\mathrm{HNC}, \mathrm{CS}, \mathrm{C}_{2} \mathrm{H}, \mathrm{H}_{2} \mathrm{CO}$ and $\mathrm{HCO}^{+}$, in addition to the ${ }^{12} \mathrm{CO},{ }^{13} \mathrm{CO}$ and $\mathrm{C}^{18} \mathrm{O}$ isotopes. Using the Keplerian disk model substantiated by the interferometer maps of $\mathrm{CO}$ or its isotopes, the higher excitation lines like $\mathrm{CN}(2-1)$ and $\mathrm{HCO}^{+}(3-2)$ provide minimal values for the $\mathrm{H}_{2}$ densities, while $\mathrm{HCN}(3-2)$ provides upper limits. Comparing this direct measurement of the mean $\mathrm{H}_{2}$ density with the measured molecular column densities, DGG were able to 1) derive directly the gas disk mass, and 2) evaluate the average molecular depletions. In DM Tau, CO is depleted by a factor 5 (refered to standard Taurus molecular cloud abundances), the other molecules being even more depleted (up to a factor 100 for $\mathrm{H}_{2} \mathrm{CO}$ ). The mass of the DM Tau gas disk is about $410^{-3} \mathrm{M}_{\odot}$, still several times smaller than the mass derived from dust emission. However, if the dust is confined within the inner $100 \mathrm{AU}$, 
molecules from this compact core would still remain undetected, despite the long integration times.

\section{Conclusions}

Most of the work presented above was based on $3 \mathrm{~mm}$ images with relatively low (2-3") angular resolution. With the recent opening of the $1.3 \mathrm{~mm}$ "window" at the IRAM interferometer, angular resolutions of $1.5^{\prime \prime}$ are routinely reached, and $0.5^{\prime \prime}$ is possible. The spectacular images of the HH 211 outflow and of the circumstellar disk of GM Aur clearly illustrate the potential gains resulting from this improvement.

The increase in imaging speed obtained from the addition of antenna 5 in 1996 also allows more objects and topics to be studied. The results presented above offer some guidelines for future projects. Without being exhaustive, let us cite a few obvious targets: proper measurements of the angular sizes of Class 0 cores, studies of more evolved outflows (Class I, II), importance of the binarity for protostellar objects, etc....

The sub-arcsecond imaging capabilities should also enable detailed comparisons with optical and near-IR images. However, all the expected benefits of such comparisons will only be realized if the absolute astrometric accuracy of optical images matches the angular resolution.

Acknowledgements: The work presented here would not have been possible without the constant effort of the IRAM technical staff in supporting the observations. We also thank the many colleagues who participate in this work, and in particular R.Bachiller, M.Simon and G.Duvert for many fruitful discussions.

\section{References}

Artimowicz P., Lubow S.H., 1994, ApJ 421, 651

Bachiller R., Guilloteau S., Dutrey A., Planesas P., Martin-Pintado R., 1995, A\&A 279 857

Beckwith S.V.W., Sargent A.I., Chini R.S., Gusten R., 1990, AJ 99, 924

Cabrit S., Guilloteau S., André P., Bertout C., Montmerle T., Schuster K., 1996, A\&A 305,527

Chandler C., Richer J., 1997, in Low Mass Star Formation - from Infall to Outflow, poster proceedings of IAU Symp. No. 182, eds. F. Malbet \& A. Castets, Observ. de Grenoble, p.76

Dutrey A., Guilloteau S., Simon M., 1994, A\&A 286, 149

Dutrey A., Guilloteau S., Duvert G., Prato L., Simon M., Schuster K., Ménard F., 1996, A\&A 309, 493

Dutrey A., Guilloteau S., Bachiller R., 1997a, A\&A in press

Dutrey A., Guilloteau S., Prato L., Simon M., Duvert G., Schuster K., Ménard F., 1997b, in preparation

Dutrey A., Guilloteau S., Duvert G., Simon M., Prato L., Ménard F., Schuster K., 1997c, in preparation 
Dutrey A., Guilloteau S., Guélin M., 1997d, A\&A 317, L55

Duvert G., Dutrey A., Guilloteau S., Ménard F., Schuster K., Prato L., Simon M., 1997, $A \& A$ in preparation

Gueth F., Guilloteau S., Viallefond F., 1995, in: Green D.A. Steffen W. (eds) YERAC95:

Proc XXVIIth Young European Radio Astronomers Conf. Cambridge Univ Press.

Gueth F., Guilloteau S., Bachiller R., 1996, A\&A 307, 891

Gueth F., Guilloteau S., Bachiller R., 1997a, A\&A in preparation

Gueth F., et al. 1997b, in preparation

Gueth F., Guilloteau S., Dutrey A., Bachiller R. 1997c, A\&A in press

Gueth F., Guilloteau S., 1997, in preparation

Guilloteau S., Bachiller R., Fuente A., Lucas R., 1992, A\&A 265, L49

Guilloteau S., Dutrey A., Guélin M., 1997, in preparation

Hayashi M., Ohashi N., Miyama S.M., 1993, ApJ 418, L71

Jensen E.L.N., Koerner D.W., Matthieu R.D., 1996, AJ 111, 2431

Lada E.A., Dutrey A., Guilloteau S., Mundy L. 1996, BAAS 189, 530

Matthieu R.D., Martin E.L., Maguzzu A., 1996, BAAS 188, 6005

McCaughrean M., O'Dell, C.R., 1996, AJ 111, 1997

McCaughrean M., Rayner J.T., Zinnecker H., 1994, ApJ 436, L189

Mundy L.G., Looney L.W., Lada E.A., 1995, ApJ 452, L137

Mundy L.G., Looney L.W., Erickson W., Grossman A., Welch W.J. Forster J.R., Wright M.C.H., Plambeck R.L., Lugten J., Thornton D.D., 1996, ApJ 464, 169

Simon M., Guilloteau S., 1992, ApJ 397, L476

Roddier C., Roddier F., Northcott M.J., Graves J.E., Jim L., 1996, ApJ 463, 326

Stapelfeldt et al., 1997, this volume. 